\title{
Distance-Engineered Plasmon-Enhanced Light Harvesting in CdSe Quantum Dots
}

\author{
Shengye Jin, ${ }^{\dagger, \S, \nabla}$ Erica DeMarco, ${ }^{\ddagger, \S, \nabla}$ Michael J. Pellin, ${ }^{\S, \perp}$ Omar K. Farha, ${ }^{\ddagger, \S}$ Gary P. Wiederrecht, ${ }^{* \dagger, \ddagger}$
} and Joseph T. Hupp ${ }^{\ddagger, \S, \perp, \#}$

${ }^{\dagger}$ Nanoscience and Technology Division, ${ }^{\perp}$ Materials Science Division, and ${ }^{\#}$ Chemical Sciences and Engineering Division, Argonne National Laboratory, Argonne, Illinois 60439, United States

${ }^{\ddagger}$ Argonne-Northwestern Solar Energy Research Center and ${ }^{\S}$ Department of Chemistry, Northwestern University, Evanston, Illinois 60208, United States

\section{Supporting Information}

ABSTRACT: Improvement of light harvesting in semiconductor quantum dots (QDs) is essential for the development of efficient QD-based solar energy conversion systems. In this study, plasmonenhanced light absorption in CdSe QDs sensitized on silver (Ag) nanoparticle (NP) films was examined as a function of interparticle (QD to Ag NP) distance. Up to 24-fold plasmonic enhancement of fluorescence from QDs was observed when the particle separation distance was $\geq 5 \mathrm{~nm}$. The enhancement effect was observed to largely
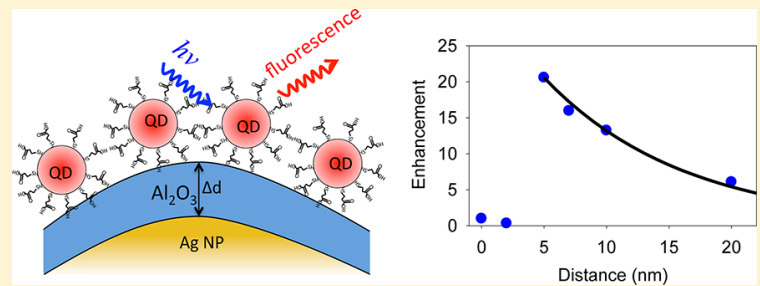
sustain the exciton lifetimes in QDs and to strongly depend on the incident photon wavelength following the plasmon resonant strength of Ag NPs, confirming that the enhanced photoluminescence was mainly due to the enhancement in photoabsorption in CdSe QDs by the plasmon of Ag NPs. This study suggests applications of Ag NPs in QD-based solar energy conversion for significantly improving light harvesting in QDs.

SECTION: Plasmonics, Optical Materials, and Hard Matter

S emiconductor quantum dots (QDs) have received extensive attention because of their attractive chemical and photophysical properties, such as ease of synthesis, tunable band gap, broad absorption spectra, large extinction coefficient, and long photogenerated exciton lifetimes. ${ }^{1-3}$ These properties make QDs a promising class of materials, potentially superior to inorganic or organic molecules, to be utilized as light-harvesting and charge-separation components in solar energy conversion schemes. ${ }^{4,5}$ For example, effective and robust photogeneration of $\mathrm{H}_{2}$ from water facilitated by semiconductor QDs (or hybrid nanostructures) with catalysts (e.g., platinum, nickel) has been previously reported in the literature. ${ }^{6-13}$ The fundamental processes in these photocatalytic reactions normally involve light harvesting by a $\mathrm{QD}$, followed by charge transfer to the catalyst, where the eventual turnover of reactants to fuels occurs. Because most photocatalytic reactions require multiple charges, effective charge accumulation is essential to realize high efficiency. This requires not only schemes with effective charge separation and suppressed charge recombination but also the development of effective approaches for the enhancement of light harvesting by QDs. Utilizing the localized surface plasmon of metal nanoparticles (NPs) or structures to increase the light harvesting in QDs can be a promising strategy to improve the efficiency in a QD-based solar energy conversion system. The localized surface plasmon resonance (LSPR) of metal particles can strongly intensify the optical field in the vicinity of the metal surfaces and thus can improve the absorption of incident photons in the light-harvesting materials nearby. ${ }^{14-17}$ This plasmonic effect has been exploited to improve efficiencies in solar cells. ${ }^{18-23}$

Although plasmon-enhanced photoluminescence in QDs by adjacent gold $(\mathrm{Au})$ or silver $(\mathrm{Ag}) \mathrm{NPs}$ or nanostructures has been broadly reported, ${ }^{24-41}$ studies focusing on plasmonenhanced light harvesting in QDs, particularly considering their applications in solar energy conversion, have rarely been described. For most previously reported examples, plasmon enhancements of QD photoluminescence were accompanied by striking decreases in exciton lifetime. ${ }^{28-38}$ Thus, metal NP/QD coupling may reduce the exciton lifetime by increasing the rate of radiative relaxation and/or by opening up energy transfer or other nonradiative relaxation pathways. ${ }^{26,31,33,34,39,42-45}$ Considering the application of QDs in solar energy conversion, a reduction of exciton lifetime may diminish the benefits of plasmon-enhanced absorption. Briefly, the shorter lifetimes could decrease yields for desired charge transfer or charge separation. They also could reduce the exciton diffusion length. Ideally, a plasmonic enhancement that improves photon absorption by QDs without significantly attenuating exciton lifetimes is preferred for applications in solar energy conversion.

Herein, we report the distance-engineered plasmonenhanced photon absorption in CdSe QDs by using Ag NPs

Received: August 22, 2013

Accepted: October 3, 2013

Published: October 3, 2013 
but without significantly shortening the QDs' exciton lifetimes. The sample configuration is shown in Figure 1. The distance

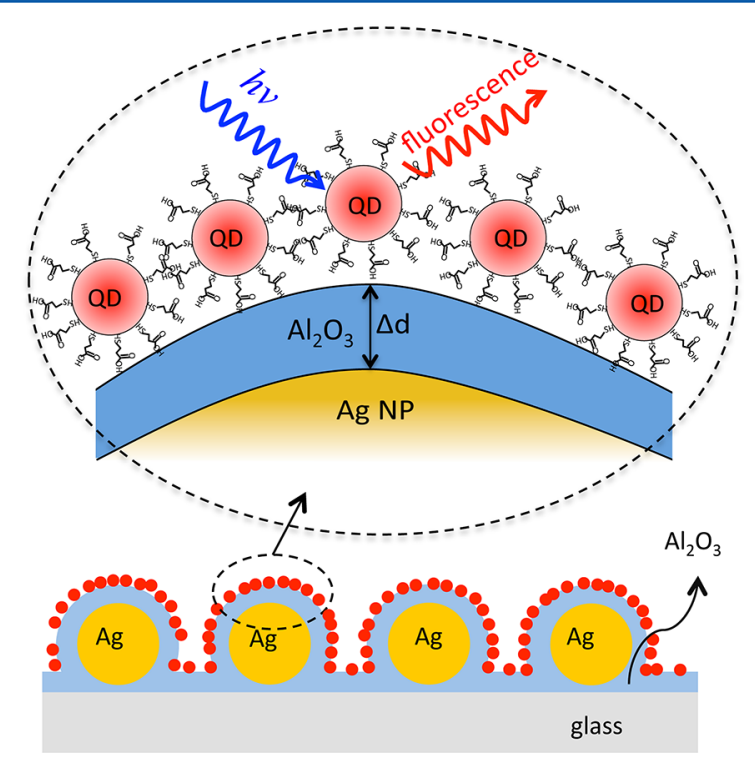

Figure 1. A schematic diagram of an $\mathrm{Al}_{2} \mathrm{O}_{3}$-coated $\mathrm{Ag} \mathrm{NP}$ film with CdSe QDs sensitized on the surface. The distance between QDs and the $\mathrm{Ag} \mathrm{NP}$ is tuned by changing the thickness $(\Delta d)$ of the $\mathrm{Al}_{2} \mathrm{O}_{3}$ layer. Upon illumination, the fluorescence and fluorescent lifetime of QDs as a function of $\mathrm{Al}_{2} \mathrm{O}_{3}$ thickness are recorded to examine the distancedependent plasmonic effect of Ag NPs.

from Ag NPs to CdSe QDs is precisely tuned by changing the thickness of the $\mathrm{Al}_{2} \mathrm{O}_{3}$ layer grown on top of $\mathrm{Ag} \mathrm{NPs}$ by atomic layer deposition (ALD). Importantly, the ALD approach produces a pinhole-free spacer layer with subnanometer control over the spacer thickness, ${ }^{46}$ which enables a precise study of plasmon-enhanced absorption by QDs as a function of chargetransfer distance and dielectric. By avoiding inhomogeneity in the spacer thickness, it has been reported that it may be possible to further increase the excitation of QDs through plasmon enhancement. ${ }^{40}$ The plasmonic effect of Ag NPs was examined by detecting the fluorescence from QDs. Both fluorescence quenching and enhancement were observed as a function of distance $\left(\mathrm{Al}_{2} \mathrm{O}_{3}\right.$ thickness). At a short distance of 2 $\mathrm{nm}$, the fluorescence of the QDs was quenched by $\sim 70 \%$ with a significantly shortened exciton lifetime, effects attributable to ultrafast energy transfer from QDs to Ag NPs. In contrast, a 5 $\mathrm{nm}$ Ag NP-to-QD distance produced dramatically plasmonenhanced fluorescence with only slightly shortened exciton lifetimes. Beyond $5 \mathrm{~nm}$, the enhancement decays exponentially with increasing separation distance. The plasmon-enhanced fluorescence of QDs can be mainly attributed to the enhanced absorption of incident photons rather than an enhanced radiative versus nonradiative time constant (photoluminescent quantum yield). This study suggests exploiting Ag NPs in QDbased solar energy conversion for significantly improving light harvesting in QDs without changing their exciton lifetimes.

The synthesis of Ag NPs with an average diameter of $40 \pm 6$ $\mathrm{nm}$ is based on a previously reported method. ${ }^{47}$ Details regarding the synthesis and the fabrication of $\mathrm{Al}_{2} \mathrm{O}_{3}$-coated $\mathrm{Ag}$ $\mathrm{NPs}$ films are in the Supporting Information (SI). The $\mathrm{Al}_{2} \mathrm{O}_{3}$ layer was grown on the top of Ag NPs by ALD. The thickness of $\mathrm{Al}_{2} \mathrm{O}_{3}$ was controlled by the number of ALD cycles. The deposited $\mathrm{Al}_{2} \mathrm{O}_{3}$ layers with 2, 5, 7, 10, and $20 \mathrm{~nm}$ thicknesses were grown using 18, 45, 64, 91, and 182 cycles, respectively. A representative AFM image of the $\mathrm{Ag} \mathrm{NP}$ film with $5 \mathrm{~nm} \mathrm{Al}_{2} \mathrm{O}_{3}$ is shown in the inset in Figure 2, indicating a uniform

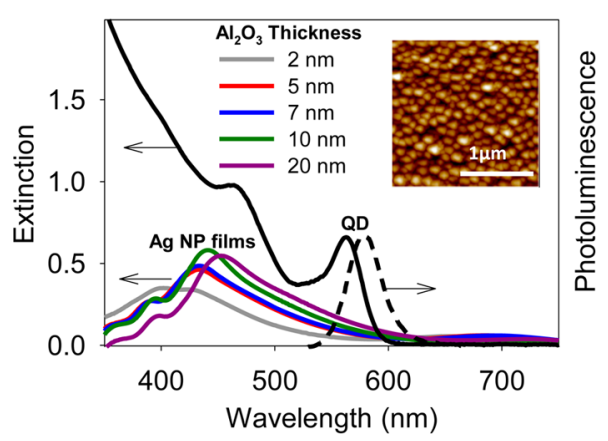

Figure 2. (a) UV-vis extinction spectra of a series of Ag NP films coated with various thicknesses $(2,5,7,10$, and $20 \mathrm{~nm})$ of $\mathrm{Al}_{2} \mathrm{O}_{3}$. Also plotted are the extinction (black solid line) and emission (black dashed line) spectra of the CdSe QDs in water. Shown in the inset is the AFM image of a $\mathrm{Ag} \mathrm{NP}$ film coated with $5 \mathrm{~nm} \mathrm{Al}_{2} \mathrm{O}_{3}$, indicating a uniform distribution of Ag NPs on the surface.

distribution of Ag NPs on the substrate ( 50 NPs per $\left.\mu \mathrm{m}^{2}\right)$. On the basis of a simple calculation, these $\mathrm{Ag}$ NPs (with $5 \mathrm{~nm}$ $\mathrm{Al}_{2} \mathrm{O}_{3}$ coating) are estimated to cause a $\sim 24 \%$ increase in the

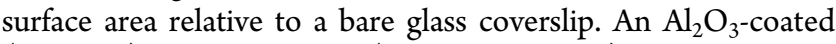
(by ALD) glass coverslip (without $\mathrm{Ag} \mathrm{NPs}$ ) was used as a control substrate (designated $\mathrm{Al}_{2} \mathrm{O}_{3}$ /glass). Water-soluble CdSe QDs, with an average diameter of $5.5 \mathrm{~nm}$, were purchased from Ocean Nanotech. The QDs were capped by 3-mercaptopropionic acid (MPA). Similar QDs have been used as sensitizers for QD-based solar cells and energy conversion processes. ${ }^{5,7,8}$ To sensitize the $\mathrm{Ag}$ NP films with QDs, the $\mathrm{Ag} / \mathrm{Al}_{2} \mathrm{O}_{3}$ films were soaked for $2 \mathrm{~h}$ in an aqueous solution of CdSe QDs having a concentration of $1.1 \mathrm{mg} / \mathrm{mL}$. The QD-sensitized Ag NP films were washed and then dried for time-resolved fluorescence detections. We note that the coverage of the QDs on the film is not directly resolved by AFM images due to the high surface heterogeneity of Ag NP films. However, the coverage can be estimated to be $\sim 50 \%$ on the surface of the substrate based on the coverage of $\mathrm{QDs}$ on $\mathrm{Al}_{2} \mathrm{O}_{3} /$ glass films under the same experimental condition (Figure S1, SI). Detection of the fluorescence from QD-covered Ag NP films was accomplished with a home-built confocal microscope system coupled with a time-correlated single photon counting (TCSPC) module. The details of the setup are described in the SI. The excitation laser (tuned from 400 to $515 \mathrm{~nm}$ ) was focused on the samples through an air objective (Olympus, $100 \times, 0.9$ N.A.), and the fluorescence from QDs was collected by the same objective and captured by the detector. The detected photons were used to construct the florescence decay curves of QDs. The collection of photons was conducted for the same length of time $(2 \mathrm{~min})$ for QDs on different substrates. During detection, the samples were rastered at a speed of $0.5 \mu \mathrm{m} / \mathrm{s}$ to avoid photodegradation of the QDs under laser excitation. Final decay curves were obtained by averaging over three to four data scans for each sample.

UV-vis extinction spectra of the Ag NP films with five $\mathrm{Al}_{2} \mathrm{O}_{3}$ thicknesses $(2,5,7,10$, and $20 \mathrm{~nm})$ are shown in Figure 2, indicating a broad spectral response region of the Ag NP plasmon. The plasmonic peak shows a red shift as the $\mathrm{Al}_{2} \mathrm{O}_{3}$ thickness increases, which is due to changes of average 
dielectric constant in the medium surrounding the $\mathrm{Ag} \mathrm{NP}$, that is, combinations of $\mathrm{Al}_{2} \mathrm{O}_{3}$ and air. The $\mathrm{UV}$-vis absorption and emission spectra of the CdSe QDs in water are shown in Figure 2. The absorption spectrum of the QDs overlaps the plasmonic region of the Ag NPs, suggesting the probability of plasmonenhanced absorption of the CdSe QDs on Ag NP films. In contrast, the emission of the CdSe QDs centered at $590 \mathrm{~nm}$ is weakly coupled with the plasmon resonance of Ag NPs and thus can be expected to undergo little extinction.

In order to examine the plasmonic effect of $\mathrm{Ag} \mathrm{NPs}$ on $\mathrm{CdSe}$ QDs, time-resolved fluorescence decays were acquired. The samples consisted of $\mathrm{QDs}$ on $\mathrm{Al}_{2} \mathrm{O}_{3}$-coated $\mathrm{Ag} \mathrm{NP}$ films with different $\mathrm{Al}_{2} \mathrm{O}_{3}$ thicknesses and a control substrate $\left(\mathrm{Al}_{2} \mathrm{O}_{3} /\right.$ glass) without Ag NPs. The decay curves are shown in Figure 3a. Because these curves were constructed by the photons collected in the same duration of times, the areas below the curves indicate the total number of emitting photons collected from the QDs during the same time (the steady-state fluorescence intensity). The steady-state fluorescence intensities (I) and the corresponding fluorescence enhancement factors (EFs) of the QDs on different substrates are calculated according to the following equations

$$
\begin{aligned}
& I=\int_{0}^{t} i(t) \mathrm{d} t \\
& \mathrm{EF}=\frac{I_{\mathrm{Ag}}}{I_{0}}
\end{aligned}
$$

where $i(t)$ is the intensity at the delay time $t$ in the decay curves and $I_{\mathrm{Ag}}$ and $I_{0}$ are the fluorescence intensities of QDs on Ag NP films and the $\mathrm{Al}_{2} \mathrm{O}_{3}$ /glass coverslip, respectively. The fluorescence intensity of QDs as a function of $\mathrm{Al}_{2} \mathrm{O}_{3}$ thickness is plotted in Figure $3 \mathrm{~b}$. The data point at zero distance/ thickness is from QDs on $\mathrm{Al}_{2} \mathrm{O}_{3}$ /glass (without $\mathrm{Ag}$ ). The $\mathrm{Al}_{2} \mathrm{O}_{3}$ layer coated on Ag NPs works as a spacer, and its thickness determines the distance from QDs to Ag NPs. Compared with the QDs on $\mathrm{Al}_{2} \mathrm{O}_{3}$ /glass, QDs on the $\mathrm{Ag} \mathrm{NP}$ films exhibit dramatic changes in fluorescence intensity as a function of the $\mathrm{Al}_{2} \mathrm{O}_{3}$ thickness. When the $\mathrm{Al}_{2} \mathrm{O}_{3}$ thickness is $2 \mathrm{~nm}$, the fluorescence of QDs is largely quenched, and the lifetime is significantly shortened compared with that for the QDs on the control substrate. However, when the thickness of the $\mathrm{Al}_{2} \mathrm{O}_{3}$ layer is increased to $5 \mathrm{~nm}$, the fluorescence of QDs is considerably enhanced compared with that on $\mathrm{Al}_{2} \mathrm{O}_{3}$ /glass. This enhancement becomes less significant when the thickness of $\mathrm{Al}_{2} \mathrm{O}_{3}$ is further increased. This enhancement in fluorescence should not be due to the increase (by only 24\%) of surface area (hence, more sensitized QDs on the surface) of Ag NP films relative to the control substrate.

The correlation between the fluorescence intensity of QDs and the thickness of the $\mathrm{Al}_{2} \mathrm{O}_{3}$ layer suggests distancedependent plasmonic effects of the Ag NPs including both fluorescence quenching and enhancement. First, when the interparticle distance is $2 \mathrm{~nm}$, the fluorescence of QDs is significantly quenched, and their lifetime is considerably shortened. Similar quenching effects for dye molecules or QDs on Au particles have been previously reported when the separation distance was less than $5 \mathrm{~nm} \cdot{ }^{39,42-44,48}$ Such quenching has been ascribed to nonradiative energy transfer from chromophores to metal NPs. Given that this quenching mechanism results in shorter decay times, we assume that a related mechanism is operative here. Another possible quenching mechanism is charge transfer from $\mathrm{QDs}$ to $\mathrm{Ag}$
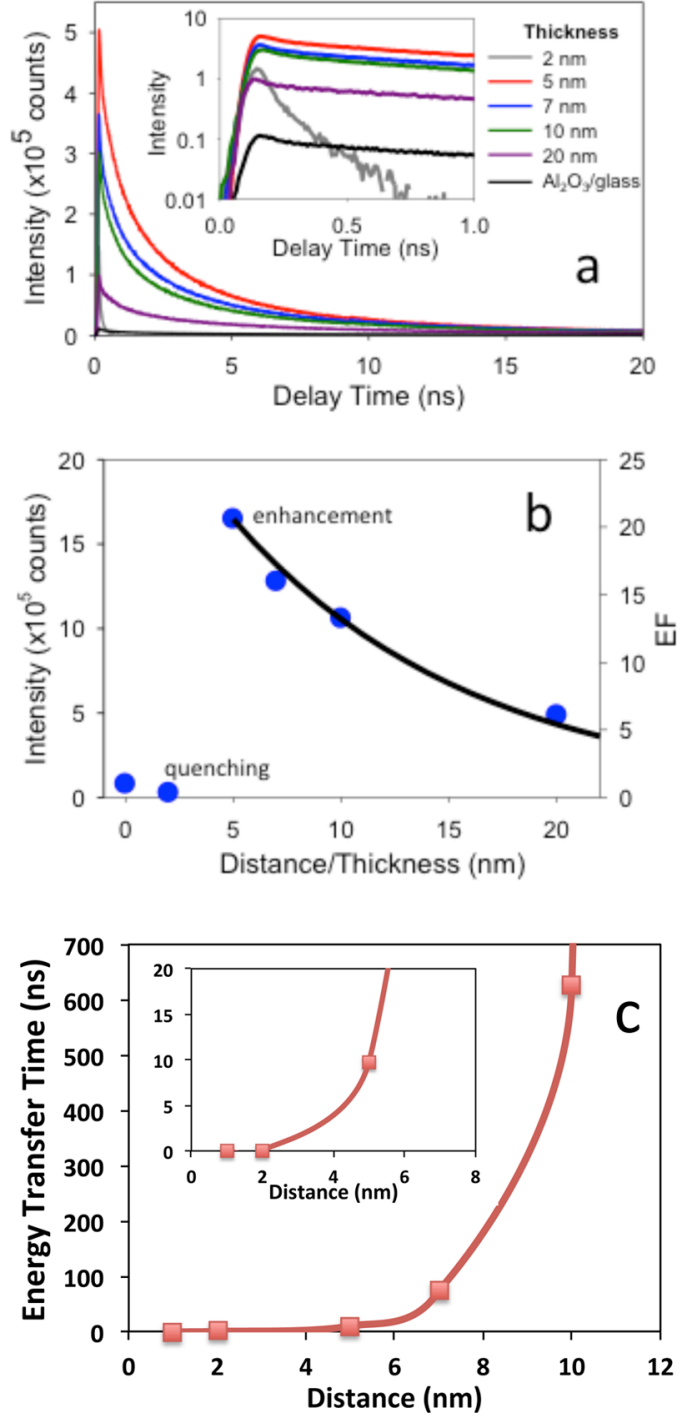

Figure 3. (a) Fluorescence decay curves of CdSe QDs sensitized on $\mathrm{Al}_{2} \mathrm{O}_{3} /$ glass (control substrate without $\mathrm{Ag} \mathrm{NPs}$ ) and $\mathrm{Al}_{2} \mathrm{O}_{3}$-coated $\mathrm{Ag}$ NP films with different $\mathrm{Al}_{2} \mathrm{O}_{3}$ thicknesses (2, 5, 7, 10, and $20 \mathrm{~nm}$ ). These curves are constructed by photons collected within the same duration of times under identical experimental conditions (excitation at $400 \mathrm{~nm}$ ). (b) The EF in fluorescence intensity of QDs as a function of distance $\left(\mathrm{Al}_{2} \mathrm{O}_{3}\right.$ thickness $)$ with an excitation at $400 \mathrm{~nm}$. The data point at $0 \mathrm{~nm}$ is from the QDs on the control substrate $\left(\mathrm{Al}_{2} \mathrm{O}_{3} /\right.$ glass $)$. The solid line is a single-exponential fit described in the text. (c) Calculated energy transfer time as a function of distance from QDs to Ag NPs based on the FRET mechanism. The details of the calculation are in the SI. The inset shows the extended view of the energy-transfer time at the short distances.

NPs. The electron-transfer time (without the spacer) from similar CdSe nanorods to Au NPs has been reported to be a few to tens of picoseconds. ${ }^{49}$ However, $\mathrm{Al}_{2} \mathrm{O}_{3}$ is an insulating material. According to the distance-dependent $\mathrm{CdSe}$ electrontransfer dynamics reported in previous literature, ${ }^{50}$ the electron-transfer time from the CdSe QD to Ag NPs at a distance of $2 \mathrm{~nm}$ can be estimated to be a few to tens of nanoseconds. Therefore, a $<80$ ps quenching time is too fast for the electron transfer with a $2 \mathrm{~nm} \mathrm{Al}_{2} \mathrm{O}_{3}$ insulator layer. We therefore conclude that this mechanism is negligible. The energy transfer from QDs to Ag NPs can follow either the surface energy transfer $(\mathrm{SET})^{51-54}$ or the Förster resonance 
energy transfer (FRET) mechanisms. ${ }^{55}$ A recent study of energy transfer from CdSe/ZnS QDs to Au NPs has indicated that the energy transfer followed the FRET mechanism when the Au NPs showed a strong LSPR. ${ }^{55}$ The energy transfer times from QDs to the Ag NP films as a function of separation distance based on the SET and FRET mechanisms are calculated (see the SI for the details) and compared in Figure S2 (SI). At a separation distance of $2 \mathrm{~nm}$, the energy-transfer times for both SET and FRET are calculated to be $<80$ ps, agreeing with the observed shortened lifetime $(<80 \mathrm{ps}$, limited by the instrument response function) of QDs on the $\mathrm{Ag} \mathrm{NP}$ film coated with $\mathrm{Al}_{2} \mathrm{O}_{3}$ of thickness $2 \mathrm{~nm}$. As the distance increases to $5 \mathrm{~nm}$, the energy-transfer times are calculated to be $2.5 \mathrm{~ns}$ for SET and $9.8 \mathrm{~ns}$ for FRET. However, the energytransfer time of $2.5 \mathrm{~ns}$ is not observed in the fluorescence decay of QDs on Ag NPs films with $5 \mathrm{~nm} \mathrm{Al}_{2} \mathrm{O}_{3}$, whose nonradiative decay time is calculated to be $\sim 8 \mathrm{~ns}$, more consistent with the energy-transfer time calculated by FRET. We therefore believe that the energy transfer from QDs to Ag NPs very likely follows the FRET mechanism. The FRET times as a function of distance are shown in Figure 3c.

As the thickness of $\mathrm{Al}_{2} \mathrm{O}_{3}$ increases, the nonradiative energytransfer rate (the FRET time from QDs to the Ag NP films) is significantly attenuated to the nanosecond time scale. As well, the QD fluorescence intensity dramatically increases to values well above that for Ag-free samples. We surmise that under these conditions, plasmonic enhancement of QD absorption now dominates nonradiative transfer. At a thickness of $5 \mathrm{~nm}$, the fluorescence enhancement reaches $\sim 24$. As the $\mathrm{Al}_{2} \mathrm{O}_{3}$ thickness increases beyond $5 \mathrm{~nm}$, the fluorescence enhancement decays slowly from this maximum value. This distance dependence is broadly consistent with those reported in the literature. $^{31,56,57}$ The correlation between fluorescence enhancement and distance can be described as an exponential decay function, which is logically close to the local density of optical states produced by the dipole in the Purcell enhancement of the plasmon's radiation rate. ${ }^{58}$ As shown in Figure $3 \mathrm{~b}$, the plasmonic fluorescence enhancement (starting from the maximum point, $F_{\max }$ at $5 \mathrm{~nm}$ ) as a function of distance $d$ $\left(\mathrm{Al}_{2} \mathrm{O}_{3}\right.$ thickness $)$ is well fit by a single-exponential function, yielding a decay parameter $D=0.89 \mathrm{~nm}^{-1}$

$$
\mathrm{EF}(d)=\mathrm{EF}_{\max } \exp (-D d) \quad(d \geq 5 \mathrm{~nm})
$$

The enhancement in fluorescence of QDs on Ag NP films can, in principle, originate from plasmonic effects on both absorption and emission. The spectral position of the plasmon resonance of Ag NPs relative to the absorption and emission spectra of QDs is determinant. ${ }^{25,27,31,36,38,59,60}$ The steady-state fluorescence intensity $(I)$ of QDs is controlled by the excitation rate $\left(k_{\text {exc }}\right)$ and the quantum yield $(\varnothing)$

$$
\begin{aligned}
& I=k_{\mathrm{exc}} \varnothing \\
& \varnothing=\frac{k_{\mathrm{r}}}{k_{r}+k_{\mathrm{nr}}}=k_{\mathrm{r}} \tau
\end{aligned}
$$

where $k_{\mathrm{r}}$ and $k_{\mathrm{nr}}$ are the radiative and nonradiative decay constants, respectively, and $\tau$ is the fluorescence lifetime. Because the absorption of the CdSe QDs overlaps well with the spectrum of the Ag NP plasmon resonance (see Figure 2), a dramatic enhancement in $k_{\text {exc }}$ in QDs is reasonably expected with the excitation at $400 \mathrm{~nm}$. Such an enhancement is due to the intensification of the local laser excitation field by Ag NPs, leading to a higher frequency of photon absorption in QDs.
Additionally, an enhancement in $k_{\mathrm{r}}$ can also occur once the emission spectrum of the luminescent species is coupled with the plasmon resonance of Ag NPs, leading to an increase in the fluorescence quantum yield and shortened fluorescence lifetime (if $k_{\mathrm{nr}}$ is not $\gg k_{\mathrm{r}}$ ). However, because the emission spectrum of the studied CdSe QDs is red-shifted by $\sim 150 \mathrm{~nm}$ relative to the extinction peak of the Ag NP plasmon film (see Figure 2), the plasmon enhancement in $k_{\mathrm{r}}$ is negligible in the currently studied system due to such a weak coupling. This conclusion is consistent with the comparison of fluorescence decay curves of QDs on different substrates, as shown in Figure S3 (SI). The decay curves show multiple exponential components. Compared with the lifetime on the control substrate $\left(\mathrm{Al}_{2} \mathrm{O}_{3} /\right.$ glass $)$, the QDs on $\mathrm{Ag} \mathrm{NP}$ films (with $\mathrm{Al}_{2} \mathrm{O}_{3}$ thickness $\geq 5 \mathrm{~nm}$ ) exhibit a slightly shortened lifetime in the slow component, while the fast component (corresponding to $\sim 70 \%$ in the amplitude) remains unchanged (see Figure S3 and Table S1 (SI) for the lifetimes of QDs on different substrates). Decay curve differences decrease as the thickness of the $\mathrm{Al}_{2} \mathrm{O}_{3}$ layer on $\mathrm{Ag}$ NPs increases. The slightly shortened slow-component lifetimes of QDs on Ag NPs compared to QDs on $\mathrm{Al}_{2} \mathrm{O}_{3}$ /glass are attributed to the presence of the energy transfer from QDs to $\mathrm{Ag}$ NPs (on a tens-hundreds of nanoseconds time scale when the distance is $\geq 5 \mathrm{~nm}$, as shown in Figure 3c) and/or the change in the dielectric constant of the substrate caused by the variation of $\mathrm{Al}_{2} \mathrm{O}_{3}$ thickness. We therefore conclude that the observed dramatic increase in fluorescence for QDs on Ag NP films primarily originates from the plasmon enhancement effect in the photoabsorption $\left(k_{\text {exc }}\right)$.

The excitation-wavelength-dependent plasmonic effect further confirms that the plasmon-enhanced fluorescence is primarily due to the enhanced photon absorption for QDs on $\mathrm{Ag}$ NPs. Besides the excitation at $400 \mathrm{~nm}$, the plasmonenhanced fluorescence at three additional excitation wavelengths $(450,490$, and $515 \mathrm{~nm})$ for $\mathrm{QDs}$ on a $\mathrm{Al}_{2} \mathrm{O}_{3}$-coated $\mathrm{Ag}$ $\mathrm{NP}$ film with a $5 \mathrm{~nm} \mathrm{Al}_{2} \mathrm{O}_{3}$ thickness was examined. (See the fluorescence decay curves of QDs at different excitation wavelengths in Figure S4, SI.) The fluorescence EF as a function of excitation wavelength is shown in Figure $4 \mathrm{~b}$. With

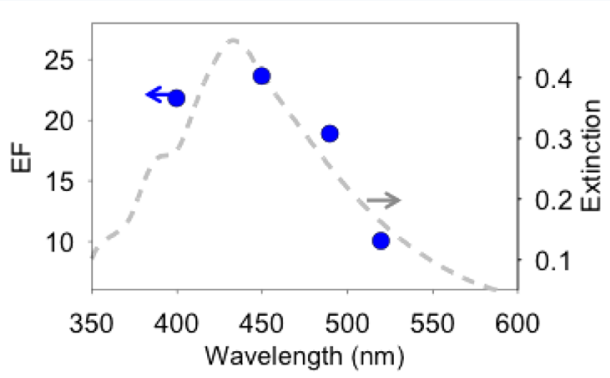

Figure 4. Fluorescence $\mathrm{EF}$ at different excitation wavelengths (blue dots) for QDs on an $\mathrm{Al}_{2} \mathrm{O}_{3}$-coated $\mathrm{Ag} \mathrm{NP}$ film with a $5 \mathrm{~nm} \mathrm{Al}_{2} \mathrm{O}_{3}$ thickness. Also shown in the panel is the extinction spectrum (dashed line) of the Ag NP film.

the excitation at $450 \mathrm{~nm}$, a slightly higher EF $(\sim 24)$ than that at $400 \mathrm{~nm}$ is achieved. However, the EF decreases to 18 and 10 , respectively, as the excitation wavelength is extended to 490 and $515 \mathrm{~nm}$. The distribution of EFs at different excitation wavelengths is consistent with the shape of the extinction spectrum associated with the Ag NP plasmon resonance. An excitation wavelength with stronger coupling to the plasmon resonance leads to a larger enhancement effect in fluorescence. 
This is consistent with the primary source of plasmon enhancement originating from increased photon absorption for the studied QDs on Ag NPs.

Plasmon enhancement of photon absorption by QDs on $\mathrm{Ag}$ NP films suggests that Ag NPs could be used to increase light harvesting by QDs for solar energy conversion. The broad and size-tunable optical spectra of QDs allow for strong (weak) coupling between the photon absorption (emission) in QDs and the plasmon resonance in Ag NPs. We have demonstrated that this coupling can lead to a dramatic enhancement in light harvesting in QDs and meanwhile, unlike in previously reported studies, maintain an unchanged photon-generated exciton lifetime with a properly tuned Ag NP-to-QD distance. We note that at a short interparticle distance $(2 \mathrm{~nm})$, the coupling between QDs and Ag NPs significantly reduced the exciton lifetime to $<80 \mathrm{ps}$, presumably by activating ultrafast energy transfer from QDs to Ag NPs. In the context of QDs as sensitizers in solar energy conversion systems, such a shortened exciton lifetime could significantly decrease overall efficiencies by decreasing quantum yields for charge transfer from QDs to catalysts or external charge acceptors. The time scales for these processes typically range from picoseconds to nanoseconds ${ }^{7,9,61-63}$ and thus diminish and even eliminate the enhancement effect in light absorption. An ideal interparticle distance from QDs to $\mathrm{Ag}$ NPs is found to be $\sim 5 \mathrm{~nm}$, at which the light absorption in QDs is enhanced by $\sim 20$-fold with only slight attenuation of the exciton lifetime.

In conclusion, we have studied the distance-engineered plasmonic effect of Ag NPs on the photophysical properties of semiconductor QDs. Both plasmon-induced fluorescence quenching and enhancement were observed as the interparticle distance from QDs to Ag NPs was tuned from 2 to $\geq 5 \mathrm{~nm}$. At 2 $\mathrm{nm}$, ultrafast energy transfer from QDs to Ag NPs was activated to quench the fluorescence of QDs. When the distance was increased to $5 \mathrm{~nm}$ or greater, the plasmonic enhancement effect became dominant, leading to an intensified fluorescence by more than 20-fold with only slightly shortened exciton lifetimes. We have confirmed that the enhanced fluorescence of QDs primarily originated from the enhancement of the local excitation field by Ag NPs. Our study indicates that with a properly tuned interparticle distance and spectral coupling between $\mathrm{QD}$ and Ag NP plasmon resonance (strong coupling in absorption but weak in emission), dramatic plasmon enhancement of photon absorption by QDs without shortening exciton lifetimes is achievable. These findings point toward the architectures necessary for efficient use of Ag NP plasmons to boost light harvesting in QD-based solar energy conversion schemes.

\section{ASSOCIATED CONTENT}

\section{(5) Supporting Information}

The details of Ag NP synthesis and ALD fabrications; experimental setup; AFM image of QDs sensitized on a glass coverslip; detailed calculation of the energy transfer rate from QDs on Ag NP films at different interparticle distances; fitting of fluorescence decays of QDs on different substrates; and fluorescence enhancement for QDs on a Ag NP film at different excitation wavelengths. This material is available free of charge via the Internet at http://pubs.acs.org.

\section{AUTHOR INFORMATION}

\section{Corresponding Author}

*E-mail: wiederrecht@anl.gov.

\section{Author Contributions}

${ }^{\nabla}$ S.J. and E.D. contributed equally to this work.

Notes

The authors declare no competing financial interest.

\section{ACKNOWLEDGMENTS}

This work was supported as part of the Argonne-Northwestern Solar Energy Research (ANSER) Center, an Energy Frontier Research Center funded by the U.S. Department of Energy, Office of Science, Office of Basic Energy Sciences, under Award Number DE-SC0001059. Use of the Center for Nanoscale Materials was supported by the U.S. Department of Energy, Office of Science, Office of Basic Energy Sciences through Contract No. DE-AC02-06CH11357.

\section{REFERENCES}

(1) Nozik, A. J.; Beard, M. C.; Luther, J. M.; Law, M.; Ellingson, R. J.; Johnson, J. C. Semiconductor Quantum Dots and Quantum Dot Arrays and Applications of Multiple Exciton Generation to ThirdGeneration Photovoltaic Solar Cells. Chem. Rev. 2010, 110, 68736890.

(2) Talapin, D. V.; Lee, J.-S.; Kovalenko, M. V.; Shevchenko, E. V. Prospects of Colloidal Nanocrystals for Electronic and Optoelectronic Applications. Chem. Rev. 2010, 110, 389-458.

(3) Kamat, P. V.; Tvrdy, K.; Baker, D. R.; Radich, J. G. Beyond Photovoltaics: Semiconductor Nanoarchitectures for Liquid-Junction Solar Cells. Chem. Rev. 2010, 110, 6664-6688.

(4) Gur, I.; Fromer, N. A.; Geier, M. L.; Alivisatos, A. P. Air-Stable All-Inorganic Nanocrystal Solar Cells Processed from Solution. Science 2005, 310, 462-465.

(5) Kamat, P. V. Quantum Dot Solar Cells. Semiconductor Nanocrystals as Light Harvesters. J. Phys. Chem. C 2008, 112, 18737-18753.

(6) Amirav, L.; Alivisatos, A. P. Photocatalytic Hydrogen Production with Tunable Nanorod Heterostructures. J. Phys. Chem. Lett. 2010, 1, $1051-1054$

(7) Zhu, H.; Song, N.; Lv, H.; Hill, C. L.; Lian, T. Near Unity Quantum Yield of Light-Driven Redox Mediator Reduction and Efficient $\mathrm{H}_{2}$ Generation Using Colloidal Nanorod Heterostructures. J. Am. Chem. Soc. 2012, 134, 11701-11708.

(8) Han, Z.; Qiu, F.; Eisenberg, R.; Holland, P. L.; Krauss, T. D. Robust Photogeneration of $\mathrm{H}_{2}$ in Water Using Semiconductor Nanocrystals and a Nickel Catalyst. Science 2012, 338, 1321-1324.

(9) Huang, J.; Mulfort, K. L.; Du, P.; Chen, L. X. Photodriven Charge Separation Dynamics in CdSe/ZnS Core/Shell Quantum Dot/ Cobaloxime Hybrid for Efficient Hydrogen Production. J. Am. Chem. Soc. 2012, 134, 16472-16475.

(10) Brown, K. A.; Dayal, S.; Ai, X.; Rumbles, G.; King, P. W. Controlled Assembly of Hydrogenase-CdTe Nanocrystal Hybrids for Solar Hydrogen Production. J. Am. Chem. Soc. 2010, 132, 9672-9680.

(11) Wen, F. Y.; Yang, J. H.; Zong, X.; Ma, B. J.; Wang, D. G.; Li, C. Photocatalytic $\mathrm{H}_{2}$ Production on Hybrid Catalyst System Composed of Inorganic Semiconductor and Cobaloximes Catalysts. J. Catal. 2011, 281, 318-324.

(12) Brown, K. A.; Wilker, M. B.; Boehm, M.; Dukovic, G.; King, P. W. Characterization of Photochemical Processes for $\mathrm{H}_{2}$ Production by CdS Nanorod-FeFe Hydrogenase Complexes. J. Am. Chem. Soc. 2012, 134, 5627-5636.

(13) Holmes, M. A.; Townsend, T. K.; Osterloh, F. E. Quantum Confinement Controlled Photocatalytic Water Splitting by Suspended CdSe Nanocrystals. Chem. Commun. 2012, 48, 371-373.

(14) Atwater, H. A.; Polman, A. Plasmonics for Improved Photovoltaic Devices. Nat. Mater. 2010, 9, 205-213.

(15) Schuller, J. A.; Barnard, E. S.; Cai, W.; Jun, Y. C.; White, J. S.; Brongersma, M. L. Plasmonics for Extreme Light Concentration and Manipulation. Nat. Mater. 2010, 9, 193-204. 
(16) Carmeli, I.; Lieberman, I.; Kraversky, L.; Fan, Z.; Govorov, A. O.; Markovich, G.; Richter, S. Broad Band Enhancement of Light Absorption in Photosystem I by Metal Nanoparticle Antennas. Nano Lett. 2010, 10, 2069-2074.

(17) Kim, I.; Bender, S. L.; Hranisavljevic, J.; Utschig, L. M.; Huang, L. B.; Wiederrecht, G. P.; Tiede, D. M. Metal Nanoparticle PlasmonEnhanced Light-Harvesting in a Photosystem I Thin Film. Nano Lett. 2011, 11, 3091-3098.

(18) Standridge, S. D.; Schatz, G. C.; Hupp, J. T. Distance Dependence of Plasmon-Enhanced Photocurrent in Dye-Sensitized Solar Cells. J. Am. Chem. Soc. 2009, 131, 8407-8409.

(19) Qi, J.; Dang, X.; Hammond, P. T.; Belcher, A. M. Highly Efficient Plasmon-Enhanced Dye-Sensitized Solar Cells through Metal@Oxide Core-Shell Nanostructure. ACS Nano 2011, 5, $7108-7116$.

(20) Rand, B. P.; Peumans, P.; Forrest, S. R. Long-Range Absorption Enhancement in Organic Tandem Thin-Film Solar Cells Containing Silver Nanoclusters. J. Appl. Phys. 2004, 96, 7519-7526.

(21) Ihara, M.; Tanaka, K.; Sakaki, K.; Honma, I.; Yamada, K. Enhancement of The Absorption Coefficient of cis-(NCS) ${ }_{2}$ Bis $\left(2,2^{\prime}\right.$ bipyridyl-4,4'-dicarboxylate)ruthenium(II) Dye in Dye-Sensitized Solar Cells by a Silver Island Film. J. Phys. Chem. B 1997, 101, 5153-5157.

(22) Wu, J.-L.; Chen, F.-C.; Hsiao, Y.-S.; Chien, F.-C.; Chen, P.; Kuo, C.-H.; Huang, M. H.; Hsu, C.-S. Surface Plasmonic Effects of Metallic Nanoparticles on the Performance of Polymer Bulk Heterojunction Solar Cells. ACS Nano 2011, 5, 959-967.

(23) Yang, J.; You, J.; Chen, C.-C.; Hsu, W.-C.; Tan, H.-r.; Zhang, X. W.; Hong, Z.; Yang, Y. Plasmonic Polymer Tandem Solar Cell. ACS Nano 2011, 5, 6210-6217.

(24) Hwang, E.; Smolyaninov, I. I.; Davis, C. C. Surface Plasmon Polariton Enhanced Fluorescence from Quantum Dots on Nanostructured Metal Surfaces. Nano Lett. 2010, 10, 813-820.

(25) Pompa, P. P.; Martiradonna, L.; Della Torre, A.; Della Sala, F.; Manna, L.; De Vittorio, M.; Calabi, F.; Cingolani, R.; Rinaldi, R. MetalEnhanced Fluorescence of Colloidal Nanocrystals with Nanoscale Control. Nat. Nanotechnol. 2006, 1, 126-130.

(26) Viste, P.; Plain, J.; Jaffiol, R.; Vial, A.; Adam, P. M.; Royer, P. Enhancement and Quenching Regimes in Metal-Semiconductor Hybrid Optical Nanosources. ACS Nano 2010, 4, 759-764.

(27) Kulakovich, O.; Strekal, N.; Yaroshevich, A.; Maskevich, S.; Gaponenko, S.; Nabiev, I.; Woggon, U.; Artemyev, M. Enhanced Luminescence of CdSe Quantum Dots on Gold Colloids. Nano Lett. 2002, 2, 1449-1452.

(28) Ma, X.; Tan, H.; Kipp, T.; Mews, A. Fluorescence Enhancement, Blinking Suppression, and Gray States of Individual Semiconductor Nanocrystals Close to Gold Nanoparticles. Nano Lett. 2010, 10, $4166-4174$

(29) Naiki, H.; Masuo, S.; Machida, S.; Itaya, A. Single-Photon Emission Behavior of Isolated CdSe/ZnS Quantum Dots Interacting with the Localized Surface Plasmon Resonance of Silver Nanoparticles. J. Phys. Chem. C 2011, 115, 23299-23304.

(30) Fu, Y.; Zhang, J.; Lakowicz, J. R. Silver-Enhanced Fluorescence Emission of Single Quantum Dot Nanocomposites. Chem. Commun. 2009, 313-315.

(31) Kuehn, S.; Hakanson, U.; Rogobete, L.; Sandoghdar, V. Enhancement of Single-Molecule Fluorescence Using a Gold Nanoparticle as an Optical Nanoantenna. Phys. Rev. Lett. 2006, 97, 017402.

(32) Masuo, S.; Naiki, H.; Machida, S.; Itaya, A. Photon Statistics in Enhanced Fluorescence from a Single CdSe/ZnS Quantum Dot in The Vicinity of Silver Nanoparticles. Appl. Phys. Lett. 2009, 95, 193106.

(33) Matsuda, K.; Ito, Y.; Kanemitsu, Y. Photoluminescence Enhancement and Quenching of Single CdSe/ZnS Nanocrystals on Metal Surfaces Dominated by Plasmon Resonant Energy Transfer. Appl. Phys. Lett. 2008, 92, 211911.

(34) Matsumoto, Y.; Kanemoto, R.; Itoh, T.; Nakanishi, S.; Ishikawa, M.; Biju, V. Photoluminescence Quenching and Intensity Fluctuations of $\mathrm{CdSe}-\mathrm{ZnS}$ Quantum Dots on an Ag Nanoparticle Film. J. Phys. Chem. C 2008, 112, 1345-1350.

(35) Munechika, K.; Chen, Y.; Tillack, A. F.; Kulkarni, A. P.; Plante, I. J.-L.; Munro, A. M.; Ginger, D. S. Spectral Control of Plasmonic Emission Enhancement from Quantum Dots near Single Silver Nanoprisms. Nano Lett. 2010, 10, 2598-2603.

(36) Shimizu, K. T.; Woo, W. K.; Fisher, B. R.; Eisler, H. J.; Bawendi, M. G. Surface-Enhanced Emission From Single Semiconductor Nanocrystals. Phys. Rev. Lett. 2002, 89, 117401.

(37) Ray, K.; Badugu, R.; Lakowicz, J. R. Metal-Enhanced Fluorescence From CdTe Nanocrystals: A Single-Molecule Fluorescence Study. J. Am. Chem. Soc. 2006, 128, 8998-8999.

(38) Song, J. H.; Atay, T.; Shi, S. F.; Urabe, H.; Nurmikko, A. V. Large Enhancement of Fluorescence Efficiency from CdSe/ZnS Quantum Dots Induced by Resonant Coupling to Spatially Controlled Surface Plasmons. Nano Lett. 2005, 5, 1557-1561.

(39) Haridas, M.; Tripathi, L. N.; Basu, J. K. Photoluminescence Enhancement and Quenching in Metal-Semiconductor Quantum Dot Hybrid Arrays. Appl. Phys. Lett. 2011, 98.

(40) Chen, Y.; Munechika, K.; Jen-La Plante, I.; Munro, A. M.; Skrabalak, S. E.; Xia, Y.; Ginger, D. S. Excitation Enhancement of CdSe Quantum Dots by Single Metal Nanoparticles. Appl. Phys. Lett. 2008, 93, 053106.

(41) Jung, D.-R.; Kim, J.; Nam, S.; Nahm, C.; Choi, H.; Kim, J. I.; Lee, J.; Kim, C.; Park, B. Photoluminescence Enhancement in CdS Nanoparticles by Surface-Plasmon Resonance. Appl. Phys. Lett. 2011, 99, 041906.

(42) Gueroui, Z.; Libchaber, A. Single-Molecule Measurements of Gold-Quenched Quantum Dots. Phys. Rev. Lett. 2004, 93, 166108.

(43) Dulkeith, E.; Morteani, A. C.; Niedereichholz, T.; Klar, T. A.; Feldmann, J.; Levi, S. A.; van Veggel, F.; Reinhoudt, D. N.; Moller, M.; Gittins, D. I. Fluorescence Quenching of Dye Molecules near Gold Nanoparticles: Radiative and Nonradiative Effects. Phys. Rev. Lett. 2002, 89, 203002.

(44) Dulkeith, E.; Ringler, M.; Klar, T. A.; Feldmann, J.; Javier, A. M.; Parak, W. J. Gold Nanoparticles Quench Fluorescence by Phase Induced Radiative Rate Suppression. Nano Lett. 2005, 5, 585-589.

(45) Acuna, G. P.; Bucher, M.; Stein, I. H.; Steinhauer, C.; Kuzyk, A.; Holzmeister, P.; Schreiber, R; Moroz, A.; Stefani, F. D.; Liedl, T.; Simmel, F. C.; Tinnefeld, P. Distance Dependence of SingleFluorophore Quenching by Gold Nanoparticles Studied on DNA Origami. ACS Nano 2012, 6, 3189-3195.

(46) Prasittichai, C.; Hupp, J. T. Surface Modification of $\mathrm{SnO}_{2}$ Photoelectrodes in Dye-Sensitized Solar Cells: Significant Improvements in Photovoltage via $\mathrm{Al}_{2} \mathrm{O}_{3}$ Atomic Layer Deposition. J. Phys. Chem. Lett. 2010, 1, 1611-1615.

(47) Evanoff, D. D.; Chumanov, G. Size-Controlled Synthesis of Nanoparticles. 1. "Silver-Only" Aqueous Suspensions via Hydrogen Reduction. J. Phys. Chem. B 2004, 108, 13948-13956.

(48) Reineck, P.; Gomez, D.; Ng, S. H.; Karg, M.; Bell, T.; Mulvaney, P.; Bach, U. Distance and Wavelength Dependent Quenching of Molecular Fluorescence by $\mathrm{Au} @ \mathrm{SiO}_{2}$ Core-Shell Nanoparticles. ACS Nano 2013, 7, 6636-6648.

(49) Sagarzazu, G.; Kohki, I.; Saruyama, M.; Sakamoto, M.; Teranishi, T.; Masuo, S.; Tamai, N. Ultrafast Dynamics and Single Particle Spectroscopy of Au-CdSe Nanorods. Phys. Chem. Chem. Phys. 2013, 15, 2141-2152.

(50) Zhu, H.; Song, N.; Lian, T. Controlling Charge Separation and Recombination Rates in CdSe/ZnS Type I Core-Shell Quantum Dots by Shell Thicknesses. J. Am. Chem. Soc. 2010, 132, 15038-15045.

(51) Chance, R. R.; Prock, A.; Silbey, R. Molecular Fluorescence and Energy Transfer near Interfaces. Adv. Chem. Phys. 1978, 37, 1-65.

(52) Persson, B. N. J.; Lang, N. D. Electron-Hole-Pair Quenching of Excited-States near a Metal. Phys. Rev. B 1982, 26, 5409-5415.

(53) Jennings, T. L.; Singh, M. P.; Strouse, G. F. Fluorescent Lifetime Quenching near $d=1.5 \mathrm{~nm}$ Gold Nanoparticles: Probing NSET Validity. J. Am. Chem. Soc. 2006, 128, 5462-5467.

(54) Yun, C. S.; Javier, A.; Jennings, T.; Fisher, M.; Hira, S.; Peterson, S.; Hopkins, B.; Reich, N. O.; Strouse, G. F. Nanometal Surface 
Energy Transfer in Optical Rulers, Breaking the FRET Barrier. J. Am. Chem. Soc. 2005, 127, 3115-3119.

(55) Li, M.; Cushing, S.; Wang, Q.; Shi, X.; Hornak, L.; Hong, Z.; $\mathrm{Wu}, \mathrm{N}$. Size-Dependent Energy Transfer between CdSe/ZnS Quantum Dots and Gold Nanoparticles. J. Phys.Chem. Lett. 2011, 2, $2125-2129$.

(56) Akbay, N.; Lakowicz, J. R.; Ray, K. Distance-Dependent MetalEnhanced Intrinsic Fluorescence of Proteins Using Polyelectrolyte Layer-by-Layer Assembly and Aluminum Nanoparticles. J. Phys. Chem. C 2012, 116, 10766-10773.

(57) Bharadwaj, P.; Novotny, L. Spectral Dependence of Single Molecule Fluorescence Enhancement. Optics Express 2007, 15, 14266-14274.

(58) Sauvan, C.; Hugonin, J. P.; Maksymov, I. S.; Lalanne, P. Theory of the Spontaneous Optical Emission of Nanosize Photonic and Plasmon Resonators. Phys. Rev. Lett. 2013, 110, 237401.

(59) Tam, F.; Goodrich, G. P.; Johnson, B. R.; Halas, N. J. Plasmonic Enhancement of Molecular Fluorescence. Nano Lett. 2007, 7, 496501.

(60) Tovmachenko, O. G.; Graf, C.; van den Heuvel, D. J.; van Blaaderen, A.; Gerritsen, H. C. Fluorescence Enhancement by MetalCore/Silica-Shell Nanoparticles. Adv. Mater. 2006, 18, 91-95.

(61) Zhu, H.; Song, N.; Lian, T. Wave Function Engineering for Ultrafast Charge Separation and Slow Charge Recombination in Type II Core/Shell Quantum Dots. J. Am. Chem. Soc. 2011, 133, 87628771.

(62) Jin, S.; Issac, A.; Stockwell, D.; Yin, F.-C.; Kindt, J.; Batista, V. S.; Robert, S.; Lian, T. Single-Molecule Interfacial Electron Transfer in Donor-Bridge-Nanoparticle Acceptor Complexes. J. Phys. Chem. B 2010, 114, 14309-14319.

(63) Jin, S. Y.; Hsiang, J. C.; Zhu, H. M.; Song, N. H.; Dickson, R. M.; Lian, T. Q. Correlated Single Quantum Dot Blinking and Interfacial Electron Transfer Dynamics. Chem. Sci. 2010, 1, 519-526. 